\title{
Helicobacter pylori infection in spouses of patients with duodenal ulcers and comparison of ribosomal RNA gene patterns
}

\author{
S D Georgopoulos, A F Mentis, C A Spiliadis ${ }^{\star}$, L S Tzouvelekis, E Tzelepi, \\ A Moshopoulos, N Skandalis
}

\begin{abstract}
Background-In recent studies, familial coinfection with the same Helicobacter pylori strains has been indicated, but more data are necessary to confirm intrafamilial spread of the micro-organism. Aims-The aim of this study was (a) to assess the frequency of $H$ pylori infection in spouses of patients with duodenal ulcers and (b) to investigate the possibility of intraspousal transmission of $H$ pylori by molecular typing of the respective strains. Patients-Sixty four patients with duodenal ulcer and their spouses were included in the study.

Methods-The $H$ pylori infection was confirmed after endoscopy by culture and histological examination of biopsy specimens, and CLO test. The isolates were compared on the basis of their rRNA gene patterns (ribopatterns) after digestion of chromosomal DNA by the restriction endonucleases HaeIII or HindIII.

Results-Of the patients, 54 were found to be $H$ pylori positive. Of the respective spouses, $42(78 \%)$ were also $H$ pylori positive. In contrast, only two out of $10(20 \%)$ partners of $H$ pylori negative patients were infected. Ribopatterns of $H$ pylori strains derived from 18 patients and their spouses showed that in each of eight couples a single strain had colonised both partners, while in the remaining 10 couples each partner was colonised by a distinct $\boldsymbol{H}$ pylori strain.

Conclusions-These data suggest person to person transmission within couples or exposure to a common source of infection.

(Gut 1996; 39: 634-638)
\end{abstract}

Keywords: Helicobacter pylori, duodenal ulcer, epidemiology, transmission, ribotyping.

Helicobacter pylori has been associated with the pathogenesis of chronic active gastritis and peptic ulcer disease. ${ }^{12}$ There is also evidence that the micro-organism is implicated in the development of gastric carcinoma. ${ }^{3}$ In spite of the numerous relevant studies, several epidemiological characteristics of the $H$ pylori infection such as the reservoirs of the microorganism and the exact mode of its transmission have not yet been clearly defined. Previous seroprevalence studies have shown that $H$ pylori infection is mainly acquired during childhood and is strongly related to socio- economic factors. ${ }^{4-6}$ However, some seropositivity occurs slowly in adults, ${ }^{5}$ suggesting that acquisition of $H$ pylori might also take place during adulthood. More details on the epidemiological features of $H$ pylori infection have been obtained by the application of molecular typing techniques, which allow efficient discrimination between $H$ pylori strains because the micro-organism exhibits an extensive genetic heterogeneity. With the aid of the above techniques, infection with the same $H$ pylori strain in people that are in close contact, such as institutionalised children and members of the same family, has been clearly demonstrated in several recent reports. ${ }^{7-10}$ The above findings, along with relevant seroepidemiological data, may indicate that a direct person to person transmission does occur and being in contact with an $H$ pylori infected person increases the risk of being infected. In this study we attempted to analyse some epidemiological aspects of $H$ pylori infection in spouses of patients with duodenal ulcer (DU) disease and compare, when available, the DNA fingerprints of the isolated $H$ pylori strains by ribotyping.

\section{Patients and methods}

\section{Study population}

From late 1992 until the end of 1994, 95 married patients with DU confirmed by endoscopy and their spouses were asked to participate. The patients were consecutive and originated from the Athens area and various parts of central and south Greece. Seventy two couples gave informed written consent in accordance with the guidelines of the Committee on Human Investigation. The following patients were excluded: (1) those with other present or recent serious diseases, or a history of intervention of the upper gastrointestinal tract, (2) those who had received antibiotics or bismuth treatment in the two months before enrolment or $\left(\mathrm{K}^{+}-\mathrm{H}^{+}\right)$-ATPase inhibitors two weeks before enrolment and (3) pregnant women. Sixty four patients with DU and their spouses fulfilled the inclusion criteria, completed a questionnaire on age, socioeconomic status, symptoms from the upper gastrointestinal tract, and the use of non-steroid antiinflammatory drugs (NSAIDs).

\section{Endoscopy and diagnosis of $\mathrm{H}$ pylori infection}

Endoscopy was performed after an overnight fast. The gastroscopes and biopsy forceps were 
thoroughly cleaned with neutral detergent and glutaraldehyde $(2.3 \%, \mathrm{v} / \mathrm{v})$ between endoscopies. A single biopsy forceps was used for each individual. Eight biopsy specimens were taken from each participant. Six were from the antrum and two from the corpus. Two of the antrum specimens and the two corpus specimens were exclusively used for histological examination, two were used for culture and two used for the CLO test. Patients were considered to be $H$ pylori positive if they produced positive results with at least two methods. Patients were considered as $H$ pylor negative if all tests gave negative results.

For histological examination, biopsy specimens were fixed in formalin and examined for the presence of $H$ pylori by a modified Giemsa staining. The Warthin-Starry technique was employed for undetermined cases.

Biopsy specimens were transported in thioglycolate broth and cultured on modified GAB-CAMP medium, ${ }^{11}$ in a microaerobic atmosphere for up to seven days. Colonies of typical appearance, Gram stain and positive for both catalase and urease production were identified as $H$ pylori.

\section{Treatment and follow up}

$H$ pylori positive patients were treated with the following regimen: omeprazole $(20 \mathrm{mg}$ twice daily) for two weeks, followed by the classic triple therapy that included hostacycline (500 $\mathrm{mg}$ four times a day), metronidazole $(500 \mathrm{mg}$ three times a day) and CBS (300 mg four times a day) for two more weeks. The $H$ pylori negative patients and their partners were used as a control population. $H$ pylori infected patients with DU who entered the study were reassessed by gastroscopy (at four weeks and 6-12 months after the completion of therapy) for ulcer healing and $H$ pylori status. The spouses of patients were subjected to one endoscopic examination, after the second endoscopy of the patient. None of the latter group received any eradication treatment no matter what the $H$ pylori status.

\section{DNA typing}

Chromosomal DNA samples of $H$ pylori isolates derived from 18 patients with DU and their spouses were prepared and partially purified by the guanidium thiocyanate method essentially as described previously. ${ }^{12}$ DNA samples were digested with restriction endonucleases HaeIII or HindIII for four hours. The digested DNA was electrophoresed at $25 \mathrm{~V}$ for 18 hours in $0.75 \%(\mathrm{w} / \mathrm{v})$ agarose. After depurination, denaturation and neutralisation, the DNA was transferred to Hybond-C nitrocellulose membranes. After prehybridisation in a mix consisting of $5 \times$ SSC $(1 \times$ SSC is $0.15 \mathrm{M}$ $\mathrm{NaCl} / 0.015 \mathrm{M}$ sodium citrate), $50 \%$ formamide, $0 \cdot 1 \%$ Ficoll, $0 \cdot 1 \%$ polyvinylpyrolidone, $0.1 \%$ bovine serum albumin, $0.5 \%$ sodium dodecyl sulphate and $500 \mathrm{mg} / \mathrm{ml}$ denatured herring sperm DNA, membranes were hybridised with a biotinylated cDNA probe that was prepared from $16+23 S$ rRNA of
Escherichia coli MRE 600 (Boehringer, Mannheim, Germany). Detection was performed using the BluGENE detection kit (Gibco-BRL, Bethesda, MD, USA).

Statistical analysis

Frequency analysis was performed by Fisher's exact test. Odds ratio was estimated using the 95\% Taylor series confidence limits.

\section{Results}

Sixty four patients (46 males) aged from 28 to 71 (mean age $46 \cdot 8$ ) years and their respective partners (46 females) aged from 25 to 68 (mean age 43.2) years were assigned to statistical analysis. Fifty four patients were $H$ pylori positive and 10 were negative. Five of the latter were chronic NSAID users. In the remaining five patients the cause of DU remained obscured. Forty two (78\%) of 54 partners of $H$ pylori positive patients were also $H$ pylori positive. In contrast, only two $(20 \%)$ spouses of $H$ pylori negative patients were $H$ pylori positive. The frequency thus of $H$ pylori infection in the spouses of $H$ pylori positive patients with DU (group A) was significantly higher than the frequency observed in the spouses of $H$ pylori negative patients with DU (group B) (odds ratio $=14.042 \cdot 26<\mathrm{OR}<$ 144.64, Fishers's exact test $\mathrm{p}<0.00084$ ). It should be noted, however, that $H$ pylor negative spouses of $H$ pylori positive patients (group A1) were significantly younger (mean age 37.4 years) than $H$ pylori positive spouses of $H$ pylori positive patients (mean age 45 years) (group A2) ( $t$ test, $\mathrm{p}<0.05$ ). Also, the duration of cohabitation was significantly longer in the latter (mean value 20.3 years) than in the former (mean value 7.4 years) group ( $t$ test, $\mathrm{p}<0.05$ ). None of the spouses of patients with DU had the disease except one who had developed duodenal erosions.

$H$ pylori strains were isolated by culture in both the patient and the partner in 26 of 42 couples $(62 \%)$. Chromosomal DNA from 36 isolates (18 from patients and 18 from the respective spouses) was analysed by comparison of the restriction pattern of the rRNA genes. Comparative analysis of the strains isolated from the remaining eight couples was not possible because of the loss of at least one strain from each pair after subculturing or during storage. Thirty isolates were successfully digested by HaeIII. Analysis of the ribopatterns of the remaining six isolates was achieved after digestion with HindIII. In Figure 1, a diagrammatic representation of the ribotype profiles of the strains examined is shown. In eight couples (44\%) both spouses harboured a single $H$ pylori strain, as it is evident from the similar ribopattern profiles (Fig 2). In the remaining 10 couples, the spouses harboured $H$ pylori strains displaying different ribopatterns. $H$ pylori ribopatterns were unique among unrelated individuals.

Overall, in six patients, relapse of DU was observed after 6-18 months. All were $H$ pylori positive on histological examination and by the 


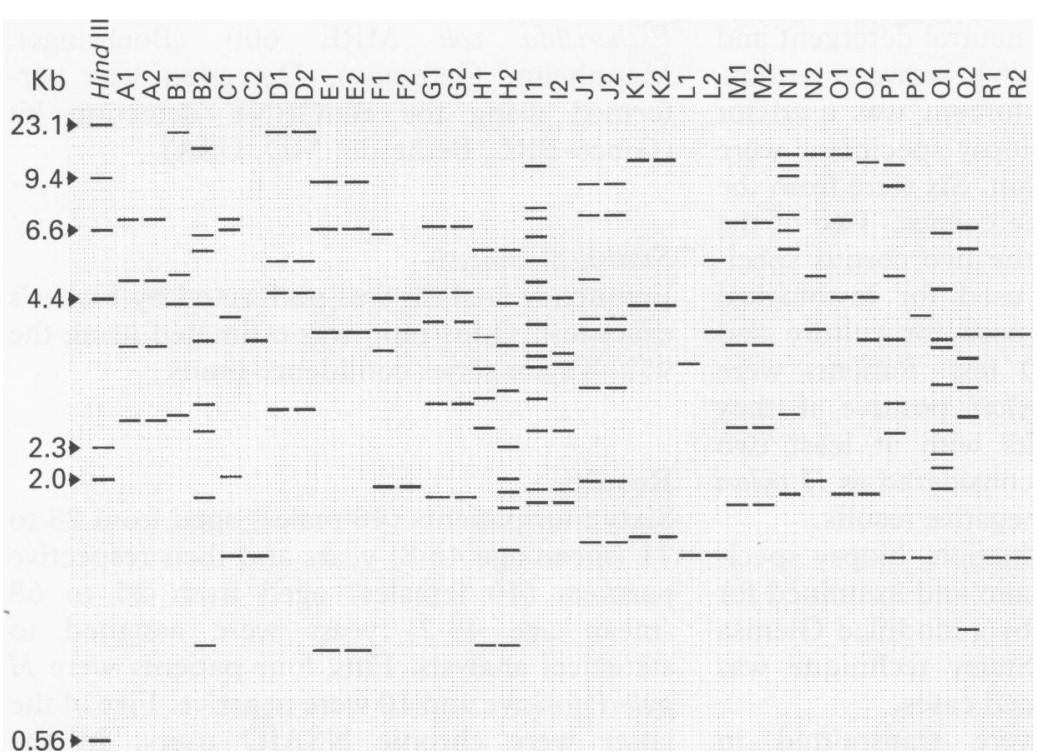

Figure 1: Diagrammatic representation of $r R N A$ gene patterns of $\mathrm{H}$ pylori strains. Each letter $(A$ to $R$ ) represents one couple, while numbers 1 and 2 represent the strains recovered from the patient with $D U$ and the respective spouse. The chromosomal DNA of $\mathrm{H}$ pylori strains from couples $A, B, D-\mathcal{F}$ and $M, N, O$ and $R$ was digested with $\mathrm{Hae} I I I$, while the $D N A$ of $\mathrm{H}$ pylori strains from couples $C, K, L, P$ and $Q$ was digested with $\mathrm{Hind} I I I$. Bacteriophage $\lambda$ DNA digested with $\mathrm{Hind} I I I$ was used as a molecular size marker. Comparisons of the ribopatterns showed that in each of the couples $A, D, E, G, \mathcal{F}, K, M$ and $R$, a single strain had colonised both partners. In the remaining 10 couples, the patients harboured $\mathrm{H}$ pylori strains displaying ribopatterns different from those of their partners' strains. that a percentage of $H$ pylori infection is acquired during adulthood. ${ }^{5}$ Since intrafamilial dissemination of $H$ pylori clones has been reported, ${ }^{7-10} 13$ we examined the possibility of intraspousal transmission of the microorganism between cohabiting married couples. In a previous seroepidemiological study it was found that as many as $70 \%$ of the adult Greek population possesses anti- $H$ pylori antibodies, ${ }^{14}$ indicating that intraspousal transmission might be difficult to demonstrate by conventional serological assays. The data presented here show a significantly higher prevalence of $H$ pylori infection in spouses of $H$ pylori positive patients with DU (group A) than in spouses of $H$ pylori negative patients (group B).

Comparison of the rRNA gene patterns of the available $H$ pylori strains showed that in 10 couples $(56 \%)$ each partner was colonised by a distinct $H$ pylori strain. Similar $H$ pylori ribopatterns were observed between spouses in each of the remaining eight couples (44\%). The high degree of the observed similarities indicated clearly that in each of these eight couples a single $H$ pylori strain had colonised both partners. It should also be mentioned that the gastric mucosa could be colonised by more than one distinct strain. ${ }^{15}$ Therefore, the percentage of couples sharing the same strain might be higher than that observed. The above results show that in a considerable percentage of couples in which one is a $H$ pylori positive patient with DU disease, there is a transfer of $H$ pylori strains or exposure to a common source of infection. However, the former notion seems to be the more likely because to date no environmental source of $H$ pylori has been recognised with certainty and the most probable ecological niche of the microorganism is the human gastric mucosa. The hypothesis of direct transfer of $H$ pylori within couples is further supported by the fact that the strain isolated in one case of relapse was similar, in terms of ribopattern, with that derived from the respective healthy spouse. Also, reinfection of patients, after successful

\section{Discussion}

$H$ pylori colonisation usually is initiated in early childhood with the highest incidence by the age of 15 years. ${ }^{4-6}$ There is also evidence suggesting
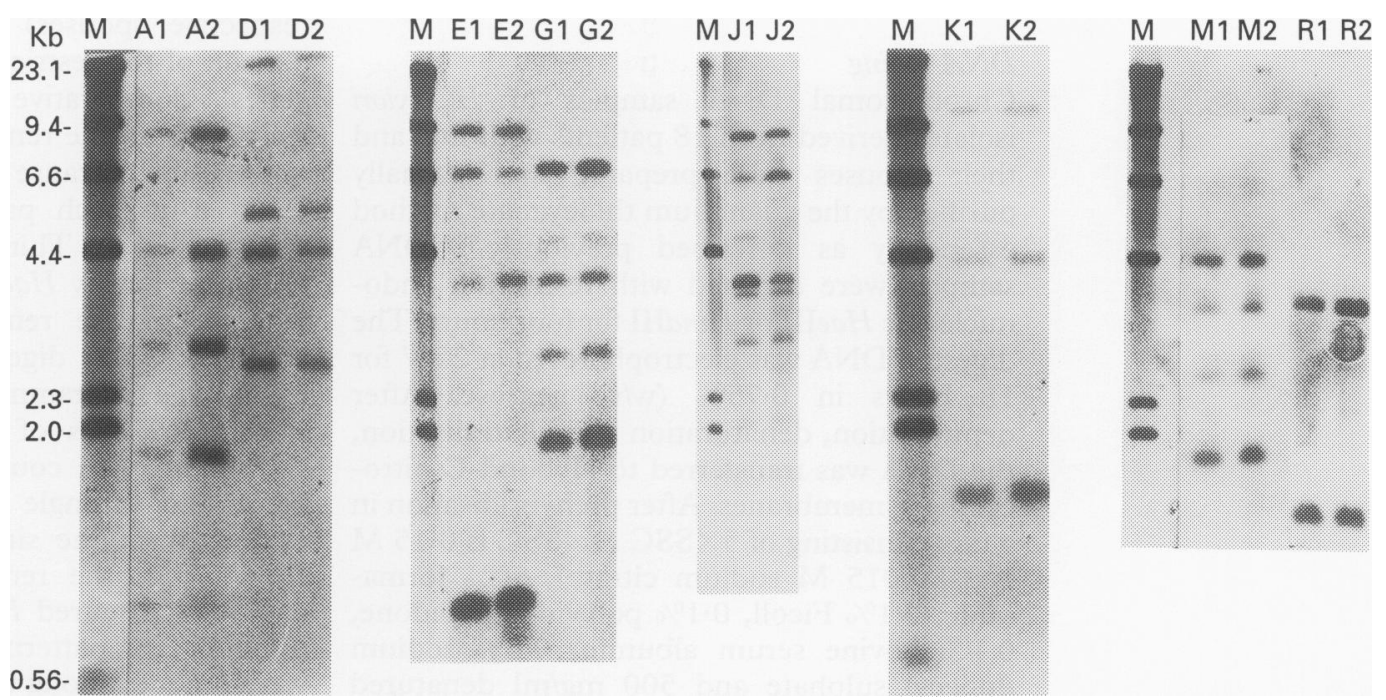

Figure 2: Ribopatterns of the $\mathrm{H}$ pylori isolates from eight patients with $D U$ (lanes: $A 1, D 1, E 1, G 1, f 1, K 1, M 1$ and R1) are presented in parallel with the ribopatterns of the similar strains (lanes: $A 2, D 2, E 2, G 2, \mathcal{F 2}, K 2, M 2$ and $R 2$ ) isolated from the respective spouses. In lanes $M, \lambda D N A$ digested with HindIII are presented. Molecular size is indicated on the left. 


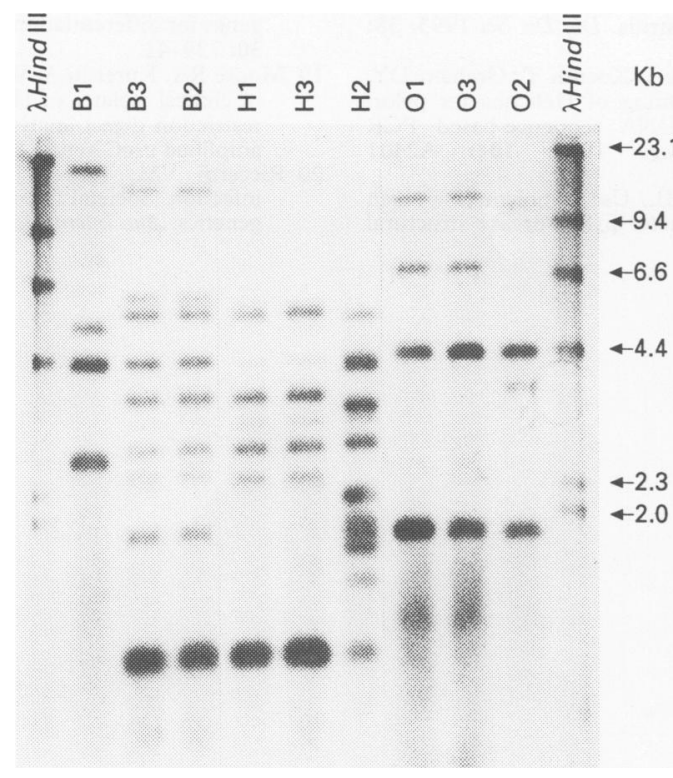

Figure 3: Comparison of $r R N A$ gene patterns of $\mathrm{H}$ pylori strains isolated from three patients who suffered a DU relapse and their spouses. In lanes $B 1, H 1$ and $O 1$, strains from the initial biopsy specimens of the patients are presented. Strains derived from either the biopsy taken after relapse or from the spouse of each patient are presented in lanes $\mathrm{B} 3, \mathrm{H} 3, \mathrm{O} 3$ and $\mathrm{B} 2, \mathrm{H} 2, \mathrm{O} 2$ respectively.

Molecular sizes are from phage $\lambda$ HindIII digests (first and last lanes). In patients $H$ and $O$, the initial strains ( $H 1$ and $O 1)$ displayed the same patterns with those isolated after relapse ( $\mathrm{H} 3$ and $\mathrm{O} 3$ ) but different from the strains isolated from their spouses ( $\mathrm{H} 2$ and $\mathrm{O} 2$ ). In patient $\mathrm{B}$, the strain isolated after DU relapse (B3) differed from the initial strain (B1), while it was similar with that of his wife (B2).

eradication therapy, by $H$ pylori strains harboured by their healthy spouses has recently been suggested. ${ }^{10}$ With respect to the direction and the mode of spread of $H$ pylori clones within couples no certain conclusions can be drawn at present. It seems reasonable to hypothesise that the actual source of infection is the $H$ pylori positive patient. As shown by histological examination, most of these patients were heavily colonised by the microorganism. Thus, mouth secretions may be contaminated from the $H$ pylori in gastric juice and the micro-organism could be transferred through the oral-oral contact of the couple. However, the faecal-oral transmission cannot be excluded. Of the eight pairs of similar $H$ pylori strains, only in one case was a subtypic difference restricted to one band of the HaeIII digestion pattern observed. The causes of the minor genetic differences between these clonal variants are unknown. They probably reflect a tendency for spontaneous genomic rearrangements or an in vivo selection of mutants.

Of interest is the fact that the $H$ pylori negative partners of $H$ pylori positive patients were younger and the duration of their cohabitation was shorter than the $H$ pylori positive partners of $H$ pylori positive patients. As already mentioned, the correlation between age and $H$ pylori infection has been established in several studies. Although it could be argued that duration of cohabitation is included in the factor 'age', it might act, at least partly, independently as a risk factor for $H$ pylori colonisation. This aspect is compatible with our finding that in eight couples the partners share the same $H$ pylori strain.

On the basis of molecular typing data, it has been suggested that there are disease specific $H$ pylori strains, ${ }^{16}{ }^{17}$ whereas other investigators report that ulcer development is irrespective of the colonising strain. ${ }^{18} 19$ The results of this study favour the latter suggestion since none of the eight spouses infected with $H$ pylori strains identical with those isolated from the respective patients with DU was dyspeptic or had signs of ulceration. Moreover, the strains isolated from three individuals who reported dyspepsia (one also had duodenal erosions) were different from the strains derived from their respective ulcerated spouses. It must be noted, however, that whether or not ulcerogenic $H$ pylori strains exist, the possibility that development of ulceration after colonisation depends, at least partly, on the genetic make up of the host. ${ }^{20}$

Overall, the data presented here support the suggestion that spouses of $H$ pylori positive patients with DU constitute a high risk group for colonisation from $H$ pylori and subsequent development of either duodenal or gastric ulcer disease. To test further this hypothesis we have initiated a follow up of the $H$ pylori positive healthy individuals detected in the present study.

1 Warren JR, Marshall B. Unidentified curved bacilli on gastric epithelium in active chronic gastritis. Lancet 1983; i: $1273-5$.

2 Graham DY. Campylobacter pylori and peptic ulcer disease. Gastroenterology 1989; 96: 614-25.

3 Logan RPH. Helicobacter pylori and gastric cancer. Lancet 1994; 344: 1078-9.

4 Mitchell HM, Li YY, Hu PJ, Liu Q, Chen M, Du GG, et al. Epidemiology of Helicobacter pylori in Southern China: identification of early childhood as the critical period for identification of early childhood as the critical

5 Banatvala N, Mayo K, Megraud F, Jennings R, Deeks JJ, Feldman RA. The cohort effect of Helicobacter pylori. $\mathcal{F}$ Infect Dis 1993; 168: 219-21.

6 Graham DY, Malaty HM, Evans DG, Evans DJ, Klein PD, Adam E. Epidemiology of Helicobacter pylori in an asymptomatic population in the United States. Gastroenterology 1991; 100: 1445-501.

7 Wang JT, Sheu JC, Lin JT, Wang TH, Wu MS. Direct DNA amplification and restriction pattern analysis of Helicobacter pylori in patients with duodenal ulcer and their families. $\mathscr{f}$ Infect Dis 1993; 168: 1544-8.

8 Bamford KB, Bickley J, Collins JSA, Johnston BT, Potts S, Boston V, et al. Helicobacter pylori: comparison of DNA fingerprints provides evidence for intrafamiliar infection. Gut 1993; 34: 1348-50.

9 Vincent P, Gottrand F, Pernes P, Husson MO, LecomteHoucke $M$, Turck $\mathrm{D}$, et al. High prevalence of Helicobacter pylori infection in cohabiting children. Epidemiology of a cluster, with special emphasis on molecular typing. Gut 1994; 35: 313-6.

10 Schutze K, Hentschel E, Dragosics B, Hirschl AM. Helicobacter pylori reinfection with identical organisms: Transmission by the patients' spouses. Gut 1995; 36: 831-2.

11 Soltesj V, Zeeberg B, Wadström T. Optimal survival of Helicobacter pylori under various transport conditions. $\mathcal{F}$ Clin Microbiol 1992; 30: 1453-6.

12 Owen RI, Hunton C, Bickley J, Moreno M, Linton D. Ribosomal RNA gene restriction patterns of Helicobacter pylori: analysis and appraisal of Hae III digests as a molecular typing system. Epidemiol Infect 1992; 109: 35-47.

13 Nwokolo CU, Bickley J, Attard AR, Owen RJ, Costas M, Fraser IA. Evidence of clonal variants of Helicobacter pylori in three generations of a duodenal ulcer disease family. Gut 1992; 33: 1323-7.

14 Pateraki E, Mentis A, Spiliadis C, Sophianos D, Stergiatou I, Skandalis N, Weir D. Seroepidemiology of Helicobacter pylori infection in Greece. FEMS Microbiol Immunol 1990; 64: 129-36.

15 Prewett EJ, Bickley J, Owen RJ, Pounder RE. DNA patterns of Helicobacter pylori isolated from gastric antrum, body, and duodenum. Gastroenterology 1992; 102: 829-33.

16 Yoshimura HH, Evans DG, Graham DY. DNA-DNA hybridisation demonstrates apparent genetic differences between Helicobacter pylori from patients with duodenal 
ulcer and asymptomatic gastritis. Dig Dis Sci 1993; 38: 1128-31.

17 Go MF, Chan KY, Versalovic J, Koeuth T, Graham DY, Lupski JR. DNA fingerprinting of Helicobacter pylori genomes with repetitive DNA sequence (REP-PCR). Gastroenterology 1993; 104: A2401 (abstract).

18 Foxall PA, Hu LT, Molbey HL. Use of polymerase chain reaction-amplified Helicobacter pylori urease structural genes for differentiation of isolates. $\mathcal{f}$ Clin Microbiol 1992; 30: 739-41.

19 Moore RA, Kureishi A, Wong S, Bryan LE. Categorization of clinical isolates of Helicobacter pylori on the basis of restriction digest analyses of polymerase chain reactionamplified ureC genes. F Clin Microbiol 1993; 31: 1334-5. 20 Riccardi VM, Rotter JI. Familial Helicobacter pylori infection. Societal factors, human genetics, and bacterial genetics. Ann Intern Med 1994; 120: 1043-4. 